\title{
Nurse interventions in right heart catheterization
}

\section{Benjamin Koren*, Jelena Mikulan}

County Hospital Čakovec, Cakovec, Croatia
KEYWORDS: nurse, right heart catheterization, monitoring

CITATION: Cardiol Croat. 2018;13(1-2):55. I https://doi.org/10.15836/ccar2018.55

*ADDRESS FOR CORRESPONDENCE: Benjamin Koren, Županijska bolnica Čakovec, I. G. Kovačića 1E, HR-40000 Čakovec, Croatia. / Phone: +385-99-7889-870 / E-mail: bencro23@gmail.com

ORCID: Benjamin Koren, https://orcid.org/0000-0002-5582-5662 • Jelena Mikulan, https://orcid.org/0000-0001-6968-6483

\section{|||||||||||||||||||||||||||||||||||||||||||||||||||||||||||||||||||||||||||||||||||||||||||||||||||||||||||||||||||||||||||||||}

The right heart catheterization (RHC) enables measurement of pressure in the right ventricle, pulmonary artery and pulmonary capillaries. It is important to take blood samples for analysis and determine oxygen saturation, which allows us to determine the intracardial level. With the help of Fick principle, it is possible to calculate the heart rate index. The catheterization is performed by the puncture of femoral, subclavian, internal jugular or cervical veins. A balloon-tipped flotation catheter-originally designed by Swan and Ganz is the most commonly used balloon catheter. The balloon tip allows the catheter to float through the right side of the hearth safely and easily. The balloon "wedges" in the distal side of catheter helps to measure the pulmonary artery pressure. Right-sided heart catheterization is indicated for patients with a history of dyspnea, valvular heart disease, or intracardiac shunts. It is most commonly used for measuring right atrium, right ventricular, pulmonary artery and pulmonary capillary pressure. The right heart catheterization is also useful for assessing pulmonary vascular resistance, tricuspidal and pulmonary function, and right ventricular pressure. ${ }^{1}$ The patient's preparation for RHC is the same as preparation for other heart catheterizations. The nurse must know the RHC protocol and must take part in the catheterization performance. It must know the normal values and appearance of the pressure curves in the heart cavities and ECG. The nurse needs to have knowledge of hemodynamic patient monitoring system and record, mark and analyze the pressure values. The nurse takes care of proper blood sampling during catheterization and proper labeling and transport of samples to the laboratory. The nurse has the skill to recognize and observe artefacts and pathological changes in vital function monitoring and timely and properly respond to them. ${ }^{2}$
RECEIVED:

February 1, 2018

ACCEPTED:

February 10, 2018

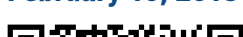

